\title{
Direct association of visit-to-visit HbA1c variation with annual decline in estimated glomerular filtration rate in patients with type 2 diabetes
}

\author{
Akiko Takenouchi', Ayaka Tsuboi', Mayu Terazawa-Watanabe², Miki Kurata ${ }^{2,3}$, Keisuke Fukuo ${ }^{1,3}$ \\ and Tsutomu Kazumi, ${ }^{3,4^{*}}$
}

\begin{abstract}
Background/Aims: This study examined associations of visit-to-visit variability of glycemic control with annual decline in estimated glomerular filtration rate (eGFR) in patients with type 2 diabetes attending an outpatient clinic.

Methods: Intrapersonal mean and coefficient of variation (CV) of 8-12 measurements of $\mathrm{HbA} 1 \mathrm{C}$ and those of 4-6 measurements of fasting and post-breakfast plasma glucose (FPG and PPG, respectively) during the first 12 months after enrollment were calculated in a cohort of 168 patients with type 2 diabetes. Annual changes in eGFR were computed using 52 (median) creatinine measurements obtained over a median follow-up of 6.0 years. Multivariate linear regressions assessed the independent correlates of changes in eGFR.

Results: $\mathrm{CV}-\mathrm{HbA} 1 \mathrm{c}$ (standardized $\beta 、-0.257 、 p=0.004$ ) were significantly and log urine albumin/creatinine ratio (standardized $\beta 、-0.155, p=0.085$ ) and smoking (standardized $\beta 、-0.186, p=0.062$ ) tended to be associated with annual eGFR decline independently of mean $\mathrm{HbA1c}$, age, sex, BMl, waist circumference, diabetes duration and therapy, means and CVs of FPG, PPG and systolic blood pressure, baseline eGFR, and uses of anti-hypertensive and lipid-lowering medications. Association between $\mathrm{HbA1c}$ variability and renal function decline was stronger in patients with albumin/creatinine ratio $\geqq 30 \mathrm{mg} / \mathrm{g}$ than in those with normoalbuminuria $(r=-0.400, p=0.003$ and $r=-0.169$, $p=0.07$, respectively).
\end{abstract}

Conclusions: Consistency of glycemic control is important to preserve kidney function in type 2 diabetic patients, in particular, in those with nephropathy.

Keywords: HbA1c, Standard deviation, Kidney function, eGFR

\section{Background}

Diabetes is an important cause of mortality and morbidity worldwide, through both direct clinical sequelae and increased mortality from cardiovascular and kidney diseases [1]. Long-term glycemic control, as expressed by hemoglobin $(\mathrm{Hb}) \mathrm{A} 1 \mathrm{c}$ levels, is the main risk factor for the development of microvascular complications including diabetic kidney disease [2, 3]. Among patients with diabetes

\footnotetext{
* Correspondence: kazumi@mukogawa-u.ac.jp

${ }^{3}$ Research Institute for Nutrition Sciences, Mukogawa Women's University, 6-46, Ikebiraki-cho, Nishinomiya, Hyogo 663-8558, Japan

${ }^{4}$ Diabetes Division, Sadamitsu Hospital, Kakogawa, Hyogo 675-0005, Japan Full list of author information is available at the end of the article
}

mellitus, elevated blood pressure (BP) is associated with progression of microvascular complications such as nephropathy and retinopathy [4]. In addition to high BP and hyperglycemia, dyslipidemia has an important role in the progression of kidney disease in patients with diabetes [5].

There is emerging interest to examine the influence of glycemic and BP variance in diabetic vascular complications [6, 7]. Recently, variation of HbA1c, a reflection of long-term glycemic fluctuation, was found to increase the risk of renal and cardiovascular complications [8-17]. In all studies on renal complications $(8-10,12-17)$, researchers focused on the relation between $\mathrm{HbA} 1 \mathrm{c}$ variability and development and/or progression of diabetic 
nephropathy. Direct association between HbA1c variability and changes in kidney function has hardly been investigated. We, therefore, asked the question whether HbA1c variability might directly associated with annual decline in estimated glomerular filtration rate (eGFR) in patients with type 2 diabetes attending a long-term follow-up in a single outpatient clinic.

\section{Methods}

The setting for this observational study was an outpatient diabetes clinic in a private hospital in Japan. Study protocol was consistent with the Japanese Government's Ethical Guidelines Regarding Epidemiological Studies in accordance with the Declaration of Helsinki. We examined a cohort of 168 patients with type 2 diabetes who had been regularly attending the clinic in 2004 and 2005. They were enrolled in the study at the first visit in 2005 and had at least 8 monthly visits with blood samplings during the first 12 months after enrollment. Of 168 patients, 153 patients (91\%) had 12 monthly visits with blood samplings. In the 153 patients, blood was withdrawn on 2 occasions; at $2 \mathrm{~h}$ after breakfast taken at home and after an overnight fasting. This was done every other month. In the remaining 15 patients, blood was obtained after an overnight fasting. The main clinical features of these subjects at baseline are reported in Table 1.

After the first visit in 2005 they were followed up in the subsequent at least 24 months through December

Table 1 Anthropometric, clinical and biochemical features of 168 patients with type 2 diabetes and correlation coefficients of annual changes in estimated glomerular filtration rate and coefficients of variation of $\mathrm{HbA} 1 \mathrm{c}$

\begin{tabular}{|c|c|c|c|c|c|c|c|}
\hline \multirow[b]{2}{*}{ Male sex $(n, \%)$} & \multicolumn{3}{|c|}{ Mean \pm SD or $n, \%$} & \multirow{2}{*}{$\frac{\triangle \mathrm{eGFR}}{-0.013}$} & & \multicolumn{2}{|l|}{$\mathrm{CV}-\mathrm{HbA1c}$} \\
\hline & 90 & , & 54 & & & -0.17 & * \\
\hline Smokers (n, \%) & 58 & , & 34 & -0.159 & $*$ & 0.111 & \\
\hline Age (years) & 62.3 & \pm & 10 & 0.037 & & -0.145 & \\
\hline $\mathrm{BMI}\left(\mathrm{kg} / \mathrm{m}^{2}\right)$ & 24.2 & \pm & 3.6 & -0.048 & & 0.045 & \\
\hline Waist circumference (cm) & 86.9 & \pm & 9.9 & -0.108 & & 0.017 & \\
\hline Duration of diabetes (years) & 9.9 & \pm & 7.3 & -0.047 & & -0.009 & \\
\hline \multicolumn{8}{|l|}{ Treatment of } \\
\hline diabetes; diet/OHA/insulin (\%) & $31 / 51 / 18$ & & & -0.078 & & 0.201 & $* *$ \\
\hline hypertension; CCB/RASi/diuretics (\%) & $34 / 41 / 5$ & & & -0.076 & & -0.044 & \\
\hline $\mathrm{HbA1c}(\%)$ & 7.0 & \pm & 0.8 & -0.050 & & 0.343 & $* * *$ \\
\hline Fasting PG (mg/dL) & 125 & \pm & 22 & -0.012 & & 0.299 & $* * *$ \\
\hline Post-breakfast PG (mg/dL) & 154 & \pm & 49 & 0.047 & & 0.229 & $* *$ \\
\hline CV-HbA1c (\%) & 7.0 & \pm & 6.4 & -0.187 & $*$ & 1 & \\
\hline CV-Fasting PG (\%) & 14.1 & \pm & 9.3 & -0.127 & & 0.473 & $* * *$ \\
\hline CV-Post-breakfast PG (\%) & 21.9 & \pm & 11.0 & -0.152 & & 0.190 & * \\
\hline Total cholesterol (mg/dL) & 188 & \pm & 21 & 0.048 & & 0.025 & \\
\hline LDL cholesterol (mg/dL) & 111 & \pm & 22 & 0.0004 & & 0.096 & \\
\hline HDL cholesterol (mg/dL) & 56 & \pm & 15 & 0.128 & & -0.202 & $* *$ \\
\hline Fasting TG (mg/dL) & 115 & \pm & 51 & -0.161 & $*$ & 0.187 & * \\
\hline Post-breakfast TG (mg/dL) & 145 & \pm & 64 & -0.164 & $*$ & 0.235 & $* *$ \\
\hline Serum creatinine (mg/dL) & 0.75 & \pm & 0.2 & -0.042 & & 0.084 & \\
\hline eGFR $\left(\mathrm{mL} / \mathrm{min} / 1.73 \mathrm{~m}^{2}\right)$ & 76 & \pm & 16 & -0.111 & & 0.165 & * \\
\hline$\triangle \mathrm{eGFR}\left(\mathrm{mL} / \mathrm{min} / 1.73 \mathrm{~m}^{2} /\right.$ year $)$ & -1.05 & \pm & 3.39 & 1 & & -0.187 & $*$ \\
\hline Uric acid (mg/dL) & 5.2 & \pm & 1.3 & -0.125 & & 0.033 & \\
\hline Systolic BP (mmHg) & 128 & \pm & 12 & -0.014 & & -0.051 & \\
\hline CV-Systolic BP (\%) & 8 & \pm & 22 & -0.035 & & 0.098 & \\
\hline Diastolic BP (mmHg) & 72 & \pm & 1 & 0.003 & & 0.112 & \\
\hline Urinary ACR (mg/g) & 84 & \pm & 322 & -0.208 & $* *$ & 0.067 & \\
\hline $\log A C R$ & 1.30 & \pm & 0.6 & -0.243 & $* *$ & 0.072 & \\
\hline
\end{tabular}

$\mathrm{OHA}$ oral hypoglycemic agents, CCB calcium channel blockers, RASi renin-angiotensin system inhibitors, PG; plasma glucose, CV; coefficient of variation, eGFR; estimated glomerular filtration rate, $\triangle \mathrm{eGFR}$; annual changes in eGFR, $B P$ blood pressure, $A C R$ albumin/creatinine ratio, $; ; p<0.05,{ }^{* *} ; p<0.01,{ }^{* * *} ; p<0.001$ 
31, 2012 to assess kidney function with a median followup of 6.0 years (interquartile range; 4.1-6.5 years). Patients with hepatitis B surface antigen or antibodies against hepatitis $C$ virus were excluded. Those who had aspartate aminotransferase and alanine aminotransferase of $100 \mathrm{U} / \mathrm{L}$ or greater, serum creatinine $\geqq 2.0 \mathrm{mg} / \mathrm{dL}$ were excluded as well. Information on smoking habits was collected through face-to-face interviews by TK. Smoking status was classified into one of three categories: current smokers, ex-smokers, and never smokers. Smokers in statistical analysis included current smokers $(n=52)$ and ex-smokers with the Brinkman index of 400 and higher $(n=5)$.

For each subject on each monthly visit, waist circumference, weight and BP were measured by registered nurses. BP using a sphygmomanometer after patients sat and rested for at least $5 \mathrm{~min}$. Plasma glucose (PG), serum lipids and lipoproteins, creatinine, hepatic enzymes, uric acid and other blood tests were measured by standard methods using an autoanalyzer. $\mathrm{HbA1C}$ values were determined by high performance liquid chromatography and inter-assay CVs were between 2.0 and $3.0 \%$. LDL cholesterol was calculated using Friedewald's formula in samples taken after an overnight fasting. Complete blood cell count was analyzed using an automated blood cell counter.

Urinary albumin was measured once during the first 3-4 months after enrollment in random urine samples using a turbidimetric immunoassay and expressed as albumin/creatinine ratio (ACR). Serum and urinary creatinine were measured enzymatically and estimated glomerular filtration rate (eGFR) was determined using the equation recommended by the Japanese Society for Nephrology [18].

Intrapersonal mean and coefficient of variation $(\mathrm{CV})$ of HbA1c, fasting and post-breakfast plasma glucose (FPG and PPG, respectively) and serum triglycerides (FTG and PTG, respectively) taken during the first 12 months after enrollment were calculated in 168 patients with type 2 diabetes; 153 patients $(91 \%)$ had 12 measurements of HbA1c, systolic BP and 6 measurements of FPG, PPG, FTG and PTG, respectively. Linear regression was used to estimate changes in eGFR using a median of 52 creatinine measurements (interquartile range; 31-60) over 6.0 years of follow-up in each patient. Baseline means of serum creatinine and eGFR in Table 1 were means of 2-4 measurements during the first 3-4 months after enrollment.

Data were presented as mean \pm SD unless otherwise stated. Differences between 2 groups were analyzed by $t$ test and frequencies of conditions by Chi-square tests. Differences among 3 groups were analyzed using analysis of variance. Correlations of annual eGFR decline and $\mathrm{CV}-\mathrm{HbA1c}$ were evaluated by Pearson correlation analysis. Stepwise multiple linear regression analyses were performed to further identify the most significant variables contributing to annual eGFR decline and CV-HbA1c. Potential confounders were forced into the model and standardized $\beta$ coefficients were calculated. The explanatory power of the model was expressed as adjusted $R^{2}$ values. A two-tailed $P<0.05$ was considered statistically significant. All calculations were performed with SPSS system 15.0 (SPSS Inc., Chicago, IL).

\section{Results}

Table 1 shows means of the intrapersonal mean values during the first 12 months after enrollment, except for age, duration of diabetes, serum creatinine, eGFR, $\triangle \mathrm{eGFR}$ and ACR. Means of age and duration of diabetes were those on enrollment of patients in the study. Baseline means of serum creatinine and eGFR in Table 1 were means of 2-4 measurements during the first 3-4 months after enrollment. ACR was measured once during the first 3-4 months after enrollment.

Patients had relatively good glycemic, lipid and BP control with a mean HbA1c of $7.0 \%$. CVs of HbA1c, FPG and PPG were $7.0 \%, 14.1 \%$ and $21.9 \%$ respectively (Table 1). Baseline eGFR averaged $76 \pm 16 \mathrm{ml} / \mathrm{min} / 1.73 \mathrm{~m}^{2}$ and eGFR change was linear and averaged $-1.05 \pm 3.39 \mathrm{ml} /$ $\mathrm{min} / 1.73 \mathrm{~m}^{2}$ per year. Among 168 patients, 27 (16.0\%) had $\mathrm{eGFR}<60 \mathrm{ml} / \mathrm{min} / 1.73 \mathrm{~m}^{2}$ and $53(31.5 \%)$ had albuminuria (microalbuminuria 47, macroalbuminuria 6).

Changes in eGFR were inversely associated with $\mathrm{CV}$ HbA1c (Fig. 1)、FTG、PTG、 log ACR and smokers (Table 1). However, eGFR changes did not show significant associations with age, sex, duration of diabetes, baseline eGFR, treatment for diabetes, mean HbA1c and mean and CV of FPG, PPG and SBP.

Multiple linear regression analysis (Table 2) revealed that CV-HbA1c (standardized $\beta 、-0.257, p=0.004$ ) were associated with and $\log$ ACR (standardized $\beta 、-0.155 、 p=$

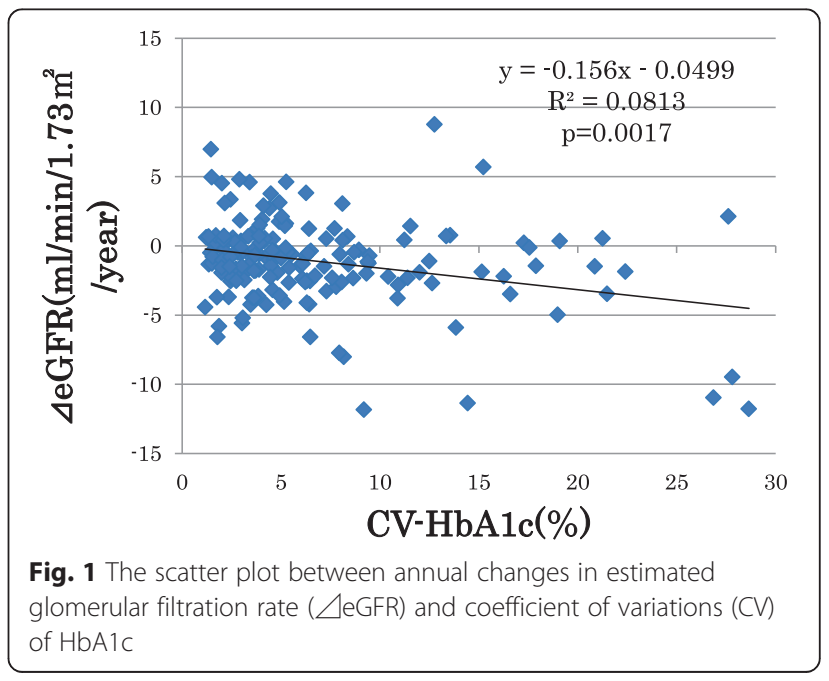


Table 2 Multiple linear regression analysis for coefficient variation of $\mathrm{HbA} 1 \mathrm{c}$ as a dependent variable

\begin{tabular}{lll}
\hline Independent variables & Standardized $\beta$ & $p$ values \\
\hline sex & -.106 & .267 \\
age & -.101 & .326 \\
BMI & .061 & .635 \\
waist circumference & -.130 & .316 \\
duration of diabetes & -.047 & .622 \\
treatment for diabetes & -.121 & .231 \\
uses of anti-hypertensive medications & .002 & .980 \\
uses of lipid-lowering medications & .055 & .537 \\
smoking & -.186 & .062 \\
Fasting PG & .084 & .488 \\
Post-breakfast PG & .106 & .376 \\
HbA1C & .027 & .812 \\
CV-HbA1C & -.257 & .004 \\
Fasting TG & -.002 & .989 \\
Post-breakfast TG & -.072 & .665 \\
log ACR & -.155 & .085 \\
eGFR & -.074 & .450 \\
\hline Abbrevations are & &
\end{tabular}

Abbreviations are the same as in Table

0.085 ) and smoking (standardized $\beta,-0.186, p=0.062$ ) tended to be associated with annual eGFR decline independently of age, sex, BMI, waist circumference, duration of and treatment for diabetes, means and CVs of FPG and PPG, mean HbA1c, baseline eGFR, FTG、PTG, and uses of anti-hypertensive and lipid-lowering medications.

Patients were divided into 3 groups according to tertiles of CV-HbA1c (Table 3). As CV-HbA1c increased, the percentage of smokers, mean $\mathrm{HbA1c}$, means and CVs of FPG and PPG, and TG increased whereas HDL cholesterol decreased. Diabetic patients in the highest as compared to the lowest and median tertiles of CV-HbA1c had faster annual decline in eGFR. Other parameters including baseline eGFR were not different among 3 groups.

Association between $\mathrm{HbA} 1 \mathrm{c}$ variability and renal function decline was significant in 53 patients with nephropathy (ACR $\geqq 30 \mathrm{mg} / \mathrm{g}$ ) but did not reach statistical significance in 115 patients without nephropathy $(\mathrm{r}=-0.400, p=0.003$ and $\mathrm{r}=-0.169, p=0.07$, respectively). Compared with patients with normoalbuminuria, annual eGFR declines were significantly faster in patients with microalbuminuria (ACR $\geqq 30 \mathrm{mg} / \mathrm{g}$ ) after controlling for confounders described above $\left(-2.0 \pm 0.4(\mathrm{SE})\right.$ vs. $-0.6 \pm 0.3 \mathrm{ml} / \mathrm{min} / 1.73 \mathrm{~m}^{2}$ per year, $p=0.01)$.

\section{Discussion}

Variations of HbA1c, a reflection of long-term glycemic fluctuation, were found to increase the risk of chronic kidney disease defines as estimated GFR (eGFR) $<60 \mathrm{ml} /$ $\mathrm{min} / 1.73 \mathrm{~m}^{2}$ in some studies in patients with type 2 diabetes [14-16]. However, we are not aware of previous studies to determine whether HbAlc variability might directly associated with annual decline rate in eGFR in patients with type 2 as well as type 1 diabetes. The present study is the first to demonstrate a direct association between CV of HbA1c and annual eGFR decline in patients with diabetes independently of mean HbA1c and known predictors of GFR decline [19]. Further, association between HbA1c variability and renal function decline was stronger in patients with nephropathy (ACR $\geqq 30 \mathrm{mg} / \mathrm{g}$ ) than in those with normoalbuminuria.

By comparison to short-term glucose variability, it has proven far less difficult to show an association between HbA1c variability and microvascular complication risk [20]. It has been shown that $\mathrm{HbA1c}$ variability predicted the development of chronic kidney disease in patients with type 2 diabetes [14, 16]. Further, Penno et al [15] have demonstrated that among 8260 patients with type 2 diabetes SD-HbA1c was associated with albuminuric chronic kidney disease independently of mean HbA1c and other known predictors of diabetic nephropathy, whereas mean HbA1c was not. These findings may be in line with our observation that albuminuria and CV-HbA1c were directly associated eGFR decline independently of mean HbA1c and other known predictors of GFR decline. However, among 4399 patients with type 2 diabetes in the intensive group of the ADVANCE trial [17], the association between SD of HbA1c and microvascular events did not reach statistical significance ( $p=0.06$ for trend) although there were significant linear associations of SD of HbA1c with combined macro-and microvascular events, major macrovascular events and all-cause mortality.

Although glycemic variability has been inconsistently associated with the risk of vascular complications in diabetes [21], several reasons may be involved in the association between visit-to-visit HbA1c variability and outcomes as suggested by Kilpatrick et al [20, 21]. They include 'metabolic memory' phenomenon [22]. They may be related to the fact that microvascular complication risk rises exponentially, rather than linearly, as HbA1c rises. They also may be related to the observation that acute improvement in HbA1c can lead to a short-term "early" worsening in retinopathy before subsequently resulting in a net long-term improvement. It is also possible that patient with $\mathrm{HbA1c}$ variability are those in whom the rest of their diabetes management is suboptimal.

Type 2 diabetic patients in the present study had annual eGFR decline which was even slower as compared with non-diabetic Japanese patients with early-stage chronic kidney disease $\left(\mathrm{eGFR}>60 \mathrm{ml} / \mathrm{min} / 1.73 \mathrm{~m}^{2}\right)$ [23] $(-1.05$ vs. $-1.64 \mathrm{ml} / \mathrm{min} / 1.73 \mathrm{~m}^{2}$ per year). Further, annual eGFR 
Table 3 Anthropometric, clinical and biochemical features of patients with type 2 diabetes according to tertiles of CV-HbA1C

\begin{tabular}{|c|c|c|c|c|c|c|c|c|c|c|}
\hline & \multicolumn{9}{|c|}{$\mathrm{CV}-\mathrm{HbA} 1 \mathrm{c}$ tertiles } & \multirow{3}{*}{$p$ values } \\
\hline & \multicolumn{3}{|l|}{ Low } & \multicolumn{3}{|c|}{ Median } & \multicolumn{3}{|l|}{ High } & \\
\hline & $(1.17-3$. & & & $(3.64-6$ & & & $(6.50-2$ & & & \\
\hline Smokers (n, \%) & 10 & , & 18.2 & 22 & ， & 39.3 & 25 & , & 44.6 & 0.008 \\
\hline Age (years) & 63.6 & \pm & 9.6 & 62.3 & \pm & 9.4 & 61.2 & \pm & 11.3 & 0.453 \\
\hline $\mathrm{BMI}\left(\mathrm{kg} / \mathrm{m}^{2}\right)$ & 24.0 & \pm & 3.9 & 24.1 & \pm & 3.0 & 24.6 & \pm & 4.0 & 0.638 \\
\hline Waist circumference (cm) & 87.2 & \pm & 9.4 & 86.6 & \pm & 8.7 & 87.0 & \pm & 11.5 & 0.959 \\
\hline Duration of diabetes (years) & 9.2 & \pm & 7.7 & 10.6 & \pm & 6.7 & 9.8 & \pm & 7.5 & 0.622 \\
\hline \multicolumn{11}{|l|}{ Treatment of } \\
\hline diabetes; diet/OHA/insulin (\%) & \multicolumn{3}{|l|}{$43 / 50 / 7$} & \multicolumn{3}{|c|}{$32 / 45 / 23$} & \multicolumn{3}{|c|}{$20 / 57 / 23$} & 0.026 \\
\hline hypertension; CCB/RASi/diuretics (\%) & \multicolumn{3}{|c|}{$36 / 39 / 5$} & \multicolumn{3}{|c|}{$30 / 41 / 4$} & \multicolumn{3}{|c|}{$38 / 45 / 5$} & 0.424 \\
\hline $\mathrm{HbA1c}(\%)$ & 6.6 & \pm & 0.6 & 7.1 & \pm & 0.7 & 7.4 & \pm & 1.0 & $<0.001$ \\
\hline Fasting PG (mg/dL) & 114 & \pm & 14 & 129 & \pm & 24 & 133 & \pm & 23 & $<0.001$ \\
\hline Post-breakfast PG (mg/dL) & 135 & \pm & 40 & 160 & \pm & 49 & 169 & \pm & 51 & 0.001 \\
\hline CV-HbA1c (\%) & 2.5 & \pm & 0.7 & 4.9 & \pm & 0.9 & 13.6 & \pm & 7.3 & $<0.001$ \\
\hline CV-Fasting PG (\%) & 8.8 & \pm & 3.9 & 14.1 & \pm & 9.7 & 19.5 & \pm & 9.6 & $<0.001$ \\
\hline CV-Post-breakfast PG (\%) & 18 & \pm & 10 & 23 & \pm & 11 & 25 & \pm & 11 & 0.007 \\
\hline Total cholesterol (mg/dL) & 188 & \pm & 19 & 189 & \pm & 18 & 187 & \pm & 25 & 0.890 \\
\hline LDL cholesterol (mg/dL) & 108 & \pm & 17 & 111 & \pm & 23 & 114 & \pm & 25 & 0.288 \\
\hline HDL cholesterol (mg/dL) & 60 & \pm & 15 & 57 & \pm & 17 & 50 & \pm & 12 & 0.001 \\
\hline Fasting TG (mg/dL) & 102 & \pm & 43 & 112 & \pm & 49 & 130 & \pm & 58 & 0.017 \\
\hline Post-breakfast TG (mg/dL) & 131 & \pm & 59 & 144 & \pm & 65 & 137 & \pm & 65 & 0.047 \\
\hline Serum creatinine $(\mathrm{mg} / \mathrm{dL})$ & 0.73 & \pm & 0.15 & 0.73 & \pm & 0.15 & 0.80 & \pm & 0.25 & 0.072 \\
\hline $\mathrm{eGFR}\left(\mathrm{mL} / \mathrm{min} / 1.73 \mathrm{~m}^{2}\right)$ & 74 & \pm & 12 & 76 & \pm & 15 & 77 & \pm & 21 & 0.641 \\
\hline$\triangle \mathrm{eGFR}\left(\mathrm{mL} / \mathrm{min} / 1.73 \mathrm{~m}^{2} /\right.$ year $)$ & -0.69 & \pm & 2.77 & -0.63 & \pm & 2.29 & -2.19 & \pm & 3.68 & 0.008 \\
\hline Uric acid (mg/dL) & 5.3 & \pm & 1.5 & 5.0 & \pm & 1.4 & 5.2 & \pm & 1.1 & 0.495 \\
\hline Systolic BP (mmHg) & 128 & \pm & 12 & 129 & \pm & 11 & 128 & \pm & 13 & 0.905 \\
\hline CV-Systolic BP (\%) & 8.2 & \pm & 2.3 & 7.6 & \pm & 2.1 & 8.3 & \pm & 2.2 & 0.254 \\
\hline Diastolic BP (mmHg) & 72 & \pm & 6 & 72 & \pm & 7 & 72 & \pm & 7 & 0.787 \\
\hline Urinary ACR (mg/g) & 21 & \pm & 24 & 69 & \pm & 152 & 162 & \pm & 532 & 0.066 \\
\hline $\log A C R$ & 1.1 & \pm & 0.5 & 1.4 & \pm & 0.6 & 1.4 & \pm & 0.7 & 0.012 \\
\hline
\end{tabular}

Mean \pm SD or $n, \%$. Abbreviations are the same as in Table 1

decline of our patients was much slower than the rate found in a previous study of Japanese type 2 diabetic patients without clinical albuminuria $\left(-2.94 \mathrm{ml} / \mathrm{min} / 1.73 \mathrm{~m}^{2}\right.$ per year) [24] despite comparable baseline eGFR (76 and $75 \mathrm{ml} / \mathrm{min} / 1.73 \mathrm{~m}^{2}$ ). These findings may be due in part to the fact that our patients had better glycemic (mean HbA1c; 7.0 vs. $8.4 \%)$ and BP (128/72 vs. $135 / 81 \mathrm{mmHg})$ control. Slower eGFR decline associated with better diabetic control in our patients may be related to failure to detect association between mean HbA1c and annual eGFR decline in the present study.

In the present study, patients with microalbuminuria had faster decline of eGFR than those with normoalbuminuria. This finding may be in line with previous studies that urinary albumin, even in the microalbuminuric range, is a predictor of renal function impairment in the general population [25], type 2 diabetic patients with preserved kidney function $[26,27]$ and in CKD patients (GFR $<50 \mathrm{ml} / \mathrm{min}$ ) [28]. In the last-cited longitudinal observational study [28], Lorenzo et al. compared the rate of renal decline in diabetic and non-diabetic CKD patients with comparable levels of albuminuria. They found that urinary ACR was a robust predictor of poor outcome. In addition, the mean slope of renal decline was similar in diabetic and non-diabetic patients when controlling for albuminuria.

The strength of the current study is that we used a 1year period when mean $\mathrm{HbAlc}$ and $\mathrm{HbA1c}$ variability were calculated from 12 measurements in $91 \%$ participants. In addition, we measured serum creatinine and 
hence eGFR during follow-up period much more frequently than in previous studies [14-16]. This could contribute to the reliability of changes in kidney function. Such a testing frequency is routine in clinical settings in Japan. However, frequent measures of $\mathrm{HbA1c}$ may artificially inflate precision and decrease standard deviation, which may impact the results. Finally, BP control and variability and postprandial TG also have been taken into accounted. Major limitations are that study participants were small in number and from a single clinic in Japan. However, the characteristics of our study participants are similar to those reported in a previous large-scale study in Japan [29].

\section{Conclusions}

The current study has shown direct association between HbA1c variability and kidney function decline in type 2 diabetic patients and demonstrated stronger association in patients with microalbuminuria than in patients with normoalbuminuria. These findings suggest that more attention should be paid by clinicians in diabetes control, avoiding excessive oscillations in blood glucose levels in type 2 diabetic patients in general and in those with microalbuminuria in particular. Further studies are needed to confirm the association in other ethnic groups with more patients.

\section{Abbreviations \\ ACR: Albumin /creatinine ratio; BP: Blood pressure; CV: Coefficient of variation; eGFR: Estimated glomerular filtration rate; FPG: Fasting plasma glucose; FTG: Fasting serum triglycerides; PPG: Post-breakfast plasma glucose; PTG: Post-breakfast serum triglycerides.}

\section{Competing interests}

The authors declare that they have no competing interests.

\begin{abstract}
Authors' contributions
AKT, AYK, MTT and MK have made substantial contributions to acquisition, analysis and interpretation of data. KF has been involved in drafting the manuscript. TK has been involved in revising it critically for important intellectual content; have given final approval of the version to be published; and agree to be accountable for all aspects of the work in ensuring that questions related to the accuracy or integrity of any part of the work are appropriately investigated and resolved. All authors read and approved the final manuscript.
\end{abstract}

\section{Acknowledgments}

The authors are indebted to all the participants for their dedicated and conscientious collaboration. We also thank Dr. Takeo Sadamitsu, MS. Ayako Kameyama and Ms. Atsuko Muranaka, Sadamitsu Hospital, for their help.

\footnotetext{
Author details

${ }^{1}$ Postgraduate School of Food Sciences and Nutrition, Nishinomiya, Japan. ${ }^{2}$ Department of Food Sciences and Nutrition, School of Human Environmental Sciences, Nishinomiya, Japan. ${ }^{3}$ Research Institute for Nutrition Sciences, Mukogawa Women's University, 6-46, Ikebiraki-cho, Nishinomiya, Hyogo 663-8558, Japan. ${ }^{4}$ Diabetes Division, Sadamitsu Hospital, Kakogawa, Hyogo 675-0005, Japan.
}

Received: 21 June 2015 Accepted: 1 September 2015

Published online: 14 September 2015

\section{References}

1. Danaei G, Lawes CM, Vander Hoorn S, Murray CJ, Ezzati M. Global and regional mortality from ischaemic heart disease and stroke attributable to higher-than-optimum blood glucose concentration: comparative risk assessment. Lancet. 2006;368:1651-9.

2. Lachin JM, Genuth S, Nathan DM, Zinman B, Rutledge BN, DCCT/EDIC Research Group. Effect of glycemic exposure on the risk of microvascular complications in the diabetes control and complications trial-revisited. Diabetes. 2008;57:995-1001.

3. Stratton IM, Adler Al, Neil HA, Matthews DR, Manley SE, Cull CA, et al. Association of glycaemia with macrovascular and microvascular complications of type 2 diabetes (UKPDS 35): prospective observational study. BMJ. 2000;321:405-12.

4. Adler Al, Stratton IM, Neil HA, Yudkin JS, Matthews DR, Cull CA, et al. Association of systolic blood pressure with macrovascular and microvascular complications of type 2 diabetes (UKPDS 36): prospective observational study. BMJ. 2000;321:412-19.

5. Rutledge JC, Ng KF, Aung HH, Wilson DW. Role of triglyceride-rich lipoproteins in diabetic nephropathy. Nat Rev Nephrol. 2010;6:361-70.

6. Nalysnyk L, Hernandez-Medina M, Krishnarajah G. Glycaemic variability and complications in patients with diabetes mellitus: evidence from a systematic review of the literature. Diabetes Obes Metab. 2010;12:288-98.

7. Parati G, Liu X, Ochoa JE. Clinical relevance of visit-to-visit blood pressure variability: impact on renal outcomes. J Hum Hypertens. 2014;28:403-9.

8. Kilpatrick ES, Rigby AS, Atkin SL. A1C variability and the risk of microvascular complications in type 1 diabetes: data from the Diabetes Control and Complications Trial. Diabetes Care. 2008;31:2198-202.

9. Wadén J, Forsblom C, Thorn LM, Gordin D, Saraheimo M, Groop PH, et al. A1C variability predicts incident cardiovascular events, microalbuminuria, and overt diabetic nephropathy in patients with type 1 diabetes. Diabetes. 2009;58:2649-55.

10. Marcovecchio ML, Dalton RN, Chiarelli F, Dunger DB. A1C variability as an independent risk factor for microalbuminuria in young people with type 1 diabetes. Diabetes Care. 2011;34:1011-23.

11. Bouchi R, Babazono T, Mugishima M, Yoshida N, Nyumura I, Toya K, et al. Fluctuations in $\mathrm{HbA} 1 \mathrm{c}$ are associated with a higher incidence of cardiovascular disease in Japanese patients with type 2 diabetes. J Diabetes Investig. 2012;3:148-55.

12. Sugawara A, Kawai K, Motohashi S, Saito K, Kodama S, Yachi Y, et al. HbA1c variability and the development of microalbuminuria in type 2 diabetes: Tsukuba Kawai Diabetes Registry 2. Diabetologia. 2012;55:2128-31.

13. Hsu CC, Chang HY, Huang MC, Hwang SJ, Yang YC, Lee YS, et al. HbA1C variability is associated with microalbuminuria development in type 2 diabetes: a 7-year prospective cohort study. Diabetologia. 2012;55:3163-72.

14. Luk AO, Ma RC, Lau ES, Yang X, Lau WW, Yu LW, et al. Risk association of $\mathrm{HbA1c}$ variability with chronic kidney disease and cardiovascular disease in type 2 diabetes: prospective analysis of the Hong Kong Diabetes Registry. Diabetes Metab Res Rev. 2013;29:384-90.

15. Penno G, Solini A, Bonora E, Fondelli C, Orsi E, Zerbini G, et al. Renal Insufficiency And Cardiovascular Events Study Group. HbA1c variability as an independent correlate of nephropathy, but not retinopathy, in patients with type 2 diabetes: the Renal Insufficiency And Cardiovascular Events (RIACE) Italian multicenter study. Diabetes Care. 2013;36:2301-10.

16. Lin CC, Chen CC, Chen FN, Li Cl, Liu CS, Lin WY, et al. Risks of diabetic nephropathy with variation in hemoglobin A1C and fasting plasma glucose. Am J Med. 2013;126:1017.

17. Hirakawa $Y$, Arima H, Zoungas S, Ninomiya T, Cooper M, Hamet $P$, et al. Impact of visit-to-visit glycemic variability on the risks of macrovascular and microvascular events and all-cause mortality in type 2 diabetes: the ADVANCE trial. Diabetes Care. 2014;37:2359-65.

18. Matsuo S, Imai E, Horio M, Yasuda Y, Tomita K, Nitta K, et al. Collaborators developing the Japanese equation for estimated GFR. Revised equations for estimated GFR from serum creatinine in Japan. Am J Kidney Dis. 2009;53:982-92.

19. Rossing K, Christensen PK, Hovind P, Tarnow L, Rossing P, Parving HH. Progression of nephropathy in type 2 diabetic patients. Kidney Int. 2004;66:1596-605.

20. Kilpatrick ES. The rise and fall of $\mathrm{HbA}(1 \mathrm{c})$ as a risk marker for diabetes complications. Diabetologia. 2012;55:2089-91.

21. Ceriello A, Kilpatrick ES. Glycemic variability: both sides of the story. Diabetes Care. 2013;36 Suppl 2:S272-5. 
22. Reddy MA, Zhang E, Natarajan R. Epigenetic mechanisms in diabetic complications and metabolic memory. Diabetologia. 2015;58:443-55.

23. Yoshida T, Takei T, Shirota S, Tsukada M, Sugiura H, Itabashi M, et al. Risk factors for progression in patients with early-stage chronic kidney disease in the Japanese population. Intern Med. 2008;47:1859-64.

24. Babazono T, Hanai K, Suzuki K, Kiuchi Y, Inoue A, Tanaka M, et al. Lower haemoglobin level and subsequent decline in kidney function in type 2 diabetic adults without clinical albuminuria. Diabetologia. 2006:49:1387-93.

25. Verhave JC, Gansevoort RT, Hillege HL, Bakker SJ, De Zeeuw D, de Jong PE, et al. An elevated urinary albumin excretion predicts de novo development of renal function impairment in the general population. Kidney Int Suppl. 2004;92:S18-21.

26. Yokoyama H, Kanno S, Takahashi S, Yamada D, Honjo J, Saito K, et al. Risks for glomerular filtration rate decline in association with progression of albuminuria in type 2 diabetes. Nephrol Dial Transplant. 2011;26:2924-30

27. Zoppini G, Targher G, Chonchol M, Ortalda V, Negri C, Stoico V, et al. Predictors of estimated GFR decline in patients with type 2 diabetes and preserved kidney function. Clin J Am Soc Nephrol. 2012;7:401-8.

28. Lorenzo V, Saracho R, Zamora J, Rufino M, Torres A. Similar renal decline in diabetic and non-diabetic patients with comparable levels of albuminuria. Nephrol Dial Transplant. 2010;25:835-41

29. Sone H, Tanaka S, limuro S, Tanaka S, Oida K, Yamasaki Y, et al. Long-term lifestyle intervention lowers the incidence of stroke in Japanese patients with type 2 diabetes: a nationwide multicentre randomised controlled trial (the Japan Diabetes Complications Study). Diabetologia. 2010;53:419-28.

\section{Submit your next manuscript to BioMed Central and take full advantage of:}

- Convenient online submission

- Thorough peer review

- No space constraints or color figure charges

- Immediate publication on acceptance

- Inclusion in PubMed, CAS, Scopus and Google Scholar

- Research which is freely available for redistribution 